\title{
EL PROBLEMA DE LA VIOLENCIA. REFLEXIONES DE SERGIO BUFANO EN LA REVISTA CONTROVERSIA
}

\section{JUAN PABLO GAUNA (UNER / CONICET / IIGG-UBA)}

Instituto de Investigaciones Gino Germani -Universidad de Buenos Aires

Pte. J. E. Uriburu 950, $6^{\circ}$ piso (Ciudad Autónoma de Buenos Aires)

jpgauna@fcedu.uner.edu.ar

\section{Resumen:}

Hacia fines de la década de 1970 los argentinos en el exilio produjeron una serie de revistas que buscaron mantener vivas distintas expresiones en el terreno de la cultura. El tono general fue el de denuncia por la violación a los derechos humanos en Argentina. No obstante se encuentra una excepción en la revista Controversia para el examen de la realidad argentina (México, 1979-1981).

En este artículo se hará un análisis de una nota publicada por Sergio Bufano, la cual servirá de precedente a los debates sobre la violencia y el lugar de las organizaciones armadas y el del Estado. Dicho escrito trata de ir a fondo en la discusión de las teorías que sustentaron las prácticas políticas emancipadoras para diferenciar la violencia de los grupos insurgentes, con relación a la ejercida por la última dictadura militar. Este derrotero lleva a un debate teórico sobre la violencia donde se cruzan el marxismo y las teorías de la cultura, el cual será introducido en este trabajo.

Palabras clave:

Controversia - Violencia - Lucha Armada - Cultura

\begin{abstract}
:
By the late 1970s the Argentine exile produced a series of magazines that sought to keep alive different expressions in the field of culture. The general tone was that of complaint for violation of human rights in Argentina, however is an exception in Controversia para el examen de la realidad argentina (Mexico, 1979-1981).

This article will analyze a note published by Sergio Bufano, which will serve as a precedent for discussions on the place of violence and armed organizations and the state. The letter tries to go deep into the discussion of the theory that supported the emancipatory to differentiate violence from insurgent groups, relative to that exerted by the military dictatorship political practices. This course takes a theoretical discussion on violence where Marxism and culture theories, which will be discussed in this work intersect.
\end{abstract}

\section{Keywords:}

Controversia - Violence - Armed Struggle - Culture 


\title{
EL PROBLEMA DE LA VIOLENCIA. REFLEXIONES DE SERGIO BUFANO EN LA REVISTA CONTROVERSIA
}

\author{
JUAN PABLO GAUNA (UNER / CONICET / IIGG-UBA)
}

jpgauna@fcedu.uner.edu.ar

Para hacer un balance de lo que fue la violencia desplegada desde las organizaciones armadas argentinas durante la década de 1970 tomaremos como referencia un artículo escrito por Sergio Bufano titulado "La violencia en Argentina: 1969-1976" que se publicó en dos partes en la revista Controversia para el examen de la realidad argentina (1979-1981). Dicha publicación se realizó en el exilio mexicano y constituyó un momento bisagra para los debates que le seguirán respecto al exilio, la derrota, la democracia, ${ }^{1}$ y lo que nos interesa aquí, la evaluación sobre la violencia durante ese período.

El texto de Bufano resulta señero, ya que entrado el siglo XXI el autor dará vida al proyecto editorial denominado Ejercitar la memoria editores. Dicha empresa retoma el espíritu de los debates en el exilio antes referidos e hizo posible, desde 2004, la publicación facsimilar de Controversia, Evita Montonera, y la revista Lucha Armada en la Argentina -con artículos que debaten desde el presente sobre violencia y política.

La perspectiva del autor en cuestión se ubica en el marco de una revisión crítica del marxismo, donde se intenta reconstruir el derrotero de las organizaciones armadas, y la violencia está puesta en relación con la transformación del sistema capitalista en Argentina. El eje central del debate propuesto por Bufano gira entorno a las estrategias llevadas adelante a través de la lucha armada, las cuales buscaron disputar el poder de Estado.

En lo que sigue, trataremos de ubicar en los textos de Controversia los considerandos respecto a la violencia que emanan del marco marxista que intenta discutir Bufano. Además nos atendremos a los parámetros de sentido propios del contexto de producción del artículo.

En paralelo propondremos otra perspectiva teórica que intentará arrojar luz sobre estas cuestiones, a saber, una visión culturalista que se hace eco del giro lingüístico, del posestructuralismo y de elementos del psicoanálisis. ${ }^{2}$ Este camino irá delineando las fronteras del marxismo a la hora de conceptualizar la violencia.

En los siguientes apartados avanzaremos sobre el artículo de Sergio Bufano, para problematizar sobre el accionar de las organizaciones armadas en la década del 1970, sobre cómo entender la violencia de ese período, e indagaremos sobre qué sucedía con la prohibición de muerte en ese entonces.

\footnotetext{
${ }^{1}$ Gago, Verónica, Controversia: una lengua del exilio, Ediciones Biblioteca Nacional, Quilmes, 2012.

2 Freud, Sigmund [1913], "El retorno infantil del totemismo", en Tótem y Tabú, Madrid, Alianza, 1985 y [1915], "Consideraciones de actualidad sobre la guerra y la muerte", en El malestar en la cultura, Madrid, Alianza, 1985. Bataille, Georges [1957], El erotismo, Barcelona, Tusquets, 1998. Girard, René [1977], La violencia y lo sagrado, Barcelona, Editorial Anagrama, 1995. Tonkonoff, Sergio, "Intercambio de tiros y tráfico de drogas: etnocriminología en Río de Janeiro", en Alegatos. Revista del Departamento de Derecho de la División de Ciencias Sociales y Humanidades $\mathrm{N}^{\circ} 58$, Azcapotzalco, Universidad Autónoma Metropolitana-Azcapotzalco, 2004 y "Mito-lógicas. La cuestión criminal en el centro de la cultura", en Mariano Gutiérrez (comp.), Populismo punitivo y justicia expresiva, Buenos Aires, Di Plácido Editores, 2011.
} 


\section{El uso de las armas como estrategia de poder}

Según Sergio Bufano lo que caracterizará a la "nueva izquierda" del período 1969-1976 será el carácter socialista de la revolución esperada y la inevitabilidad de la violencia como método de acceso al poder. Todo esto sin mediaciones democrático-burguesas, ubicando a la Argentina como un país capitalista dependiente. "Se trata de proyectos que rompen con el reformismo, con el estatismo stalinista, y que fundamentalmente reinterpretan (...) la actuación que le cupo a la izquierda desde 1943 en adelante."’3

Teniendo en cuenta que la violencia de ese período fue ejercida desde el Estado hacia las distintas prácticas de las organizaciones populares, el interrogante que se presenta es de qué violencia se trató y qué la diferencia de la llevada adelante por las organizaciones armadas.

Una de las cuestiones que advierte Bufano como problemática en el artículo que nos ocupa, es la base social de las organizaciones armadas, preeminentemente universitaria y con pocos puntos de acercamiento con la clase obrera, mayormente peronista; entonces... ¿qué será lo distintivo de esta experiencia y qué estrategia se llevaría adelante?:

"La violencia es asumida como proyecto estratégico para el acceso del pueblo al poder; ése es, precisamente, uno de los saltos cualitativos de estas nuevas formaciones políticas con respecto al reformismo de sus troncos originarios. Pero la estrategia es concebida como la culminación de un largo proceso de sucesivos pasos tácticos en los cuales las masas madurarán paulatinamente su conciencia revolucionaria de clase." ${ }^{4}$

Queda en evidencia que la violencia sería un camino que conduce al poder y luego sobrevendrían las grandes transformaciones de la mano de un cambio de conciencia de clase acorde a la revolución.

Bufano señala la importancia de la conformación de un sujeto revolucionario que fue olvidado por las organizaciones armadas. La serie de fracasos de los intentos foquistas es un ejemplo de ello.

"Rápidamente y con lujo de violencia por parte de los ejércitos esos proyectos irán fracasando o quedarán, como en el caso de Colombia, completamente aislados de las luchas sociales. La culminación de este reflujo será la muerte de Guevara en Bolivia, el giro de Cuba en sus propuestas hacia el continente y el cuestionamiento de las tesis de Debray." ${ }^{5}$

No obstante se avanzará en la incorporación de la violencia a las prácticas políticas y en la asunción del protagonismo por parte de la vanguardia en el proceso que llevará al camino de la revolución. La potencia de fuego y el espontaneísmo de masas irán marcando el camino, cuestión que Bufano dará por superada en los '60, pero que persistirá en la década de 1970 -ejemplos de esto lo constituyen distintas operaciones de Montoneros, como el secuestro del cónsul honorario de Estados Unidos, John Patrick Egan, en febrero de 1975; o la "operación monstruo" contra el Ejército en ese mismo año. ${ }^{6}$

El primer artículo de Bufano concluye con la referencia a las experiencias armadas en Brasil y Uruguay que influyeron a las distintas tendencias argentinas, las cuales se debatían entre:

\footnotetext{
3 Bufano, Sergio, "La violencia en Argentina: 1969-1976", en revista Controversia para el examen de la realidad argentina $\mathrm{N}^{\circ} 1,1979$, p. 16.

${ }^{4}$ Bufano, Sergio, "La violencia en Argentina...", op. cit., Controversia $\mathrm{N}^{\circ} 1$, p. 16.

${ }^{5}$ Bufano, Sergio, "La violencia en Argentina...", op. cit., Controversia No 1, p. 16.

${ }^{6}$ Gillespie, Richard [1982], Soldados de Perón: historia crítica sobre los Montoneros, Villa Ballester, Sudamericana, 2011.
} 
“(...) cuál será la línea de masas a seguir y cuál la estrategia, ya sea de guerra prolongada o insurrección popular. Dictadura del proletariado o gobierno obrero y popular forman parte de esa polémica que tiene raíces muy profundas en la militancia argentina., ${ }^{\prime 7}$

Las estrategias a seguir se desarrollarán en la segunda parte del artículo en cuestión y se avanzará en el análisis de la escalada armada.

\section{Entre la lucha de clases y el retorno de lo abyecto}

Bufano señala que a partir de 1969 hay un período de alza en la violencia. Ese año marcó el paso hacia una lucha militar que sacó a las organizaciones insurgentes del ámbito universitario en el marco de una agudización de las contradicciones sociales. El mismo se caracteriza por el surgimiento de la violencia organizada y espontánea.

El autor describe el período de este modo:

"En Córdoba, Rosario, Tucumán, Corrientes se aprecian formas de lucha masivas e inéditas hasta ese momento que enfrentan a los sectores revolucionarios ante la alternativa política -y también militar- de dirigir o no esa tendencia social."

Esa energía social que se expresaba bajo distintas formas contra la dictadura militar de Onganía y el orden establecido es leída desde el marxismo como fruto de la maduración de la conciencia de clase y del incremento de las contradicciones fundamentales en el marco capitalista de ese entonces. Ahora bien, la pregunta que surge en consecuencia es hasta qué punto la conducción de la violencia se da en forma racional y planificada.

Veamos cómo continúa el desarrollo del redactor de Controversia:

"La violencia, que históricamente aparece como el recurso natural de resolución de las diferencias de clases, comienza a integrarse como una metodología más en las luchas populares. En sus inicios como producto de la acción de reducidas formaciones sociales; posteriormente -y sin duda en forma parcial-, será asumida por los sectores más avanzados del campo popular."

Sobre la cuestión de cómo se integró la violencia a las luchas populares se discute álgidamente en la revista en cuestión, poniéndose en el tapete el problema de la jerarquización de la violencia y la diferenciación a que da lugar la misma. La jerarquización remite directamente a la militarización de la violencia, y ya en el marco de las organizaciones armadas, el ingreso de la misma en un juego de estrategias y de cálculos. En el caso de la diferenciación, ella supondrá en última instancia el encapsulamiento de las organizaciones insurgentes, el paso a la clandestinidad y la ruptura total con sectores populares que apoyaban su empresa.

Sobre la adhesión de las masas, las organizaciones armadas desplegaron diversas acciones, pero ninguna permitió -en términos de Bataille-adquirir categoría, prestigio, o gloria duraderos. Todo esto no se confunde ni con la fuerza -cuestión sobrevalorada por las organizaciones armadas-, ni con el derecho.

La actividad de las formaciones político-militares durante los primeros años evitó las muertes. Las masas debían acostumbrarse progresivamente a la violencia organizada. Así lo reseña Bufano:

\footnotetext{
${ }^{7}$ Bufano, Sergio, “La violencia en Argentina...”, op. cit., Controversia $\mathrm{N}^{\circ} 1$, p. 17.

${ }^{8}$ Bufano, Sergio, "La violencia en Argentina: 1969-1976", en revista Controversia para el examen de la realidad argentina $\mathrm{N}^{\circ} 2-3,1979$, p. 10.

${ }^{9}$ Bufano, Sergio, “La violencia en Argentina...", op. cit., Controversia $\mathrm{N}^{\circ}$ 2-3, p. 10.
} 
"Se trata de brindar una imagen que no esté asociada a la muerte y sí con la justicia popular; simultáneamente, se trata de demostrar que la hegemonía de la violencia, que siempre se reservó la clase dominante, puede ser disputada.

"Hasta 1972 la relación vanguardia-masas se mantiene a través de la propaganda."10

Las formaciones político-militares se movieron entre la simpatía del pueblo y como un grupo de presión. La dictadura parecía retroceder y la sociedad civil apoyó la lucha armada que vio acrecentado su poder. Pero el puente hacia los obreros no se pudo construir, como tampoco la integración de los habitantes de las villas -beneficiarios de algunas acciones de las organizaciones armadas.

La violencia armada no fue de la naturaleza que el marxismo esperaba. Así, la lucha no se daba en el marco de la tan mentada lucha de clases, sino que la misma era como una especie de residuo persistente, al cual la dictadura no pudo poner en caja. La proscripción del peronismo y todo lo que ello aparejaba fue claramente un caldo de cultivo para la insurrección, de manera que si no se integraba a dicha fuerza a la vida pública la radicalización de la violencia continuaría. Así ocurrió, y el Gral. Alejandro Lanusse tuvo que llamar a elecciones. No obstante esto, cierta inercia ya estaba en marcha en las organizaciones armadas, y por la dinámica de los acontecimientos será difícil de detener. Por eso Bufano da cuenta de la figura del hombre aparato y hacia dónde fue conducida la violencia:

"Esa desarticulación entre lo político y lo militar dio lugar a una escisión que no siempre se tradujo en fracturas; por el contrario, ambos sectores [los de la acción armada y el partido] convivieron en las mismas organizaciones, aunque el hombre de aparato fue desarrollando una concepción que ganó espacio interno y confundió disciplina con la democracia, la seguridad con el aislamiento, la base social con el refugio guerrillero y, lo que sin duda alguna fue lo más grave, la ofensiva militar con la ofensiva de masas."11

El autor señala lo grave que fue confundir la tolerancia de la violencia y su legitimidad con un estado de revolución en el cual se podía disputar el poder al Estado, además: “(...) el desplazamiento del centro de gravedad a lo militar se tradujo en un empobrecimiento teórico y político de los cuadros formados la década anterior." ${ }^{\text {"2 }}$

\section{La violencia en el período democrático 1973-1976}

Cuando asumió el gobierno democrático en mayo de 1973 las organizaciones armadas contaban con un poder de fuego como nunca antes pero, refiere Bufano, quedaron sin capacidad de lectura de los acontecimientos políticos de ese entonces.

En cuanto a la naturaleza de la violencia de la época, el autor señala lo siguiente:

"La violencia que se ha entronizado en toda la estructura de la sociedad civil no es producto de la voluntad subjetiva de sectores marginales, sino que ocupa un lugar destacado en todas las relaciones políticas y sociales. Las ocupaciones de fábrica, escuelas, organismos gubernamentales y sindicatos se realizan con armas (...)."13

En ese contexto había una pugna por establecer un poder que esté por encima de toda la sociedad. Bufano pone esta puja en términos de lucha de clases y como escenario clave el enfrentamiento armado en Ezeiza para recibir a Juan Domingo Perón.

\footnotetext{
${ }^{10}$ Bufano, Sergio, "La violencia en Argentina...", op. cit., Controversia $\mathrm{N}^{\circ} 2-3$, p. 10.

${ }^{11}$ Bufano, Sergio, "La violencia en Argentina...", op. cit., Controversia $\mathrm{N}^{\circ}$ 2-3, p. 10.

${ }^{12}$ Bufano, Sergio, "La violencia en Argentina...", op. cit., Controversia $\mathrm{N}^{\circ} 2-3$, p. 10.

${ }^{13}$ Bufano, Sergio, "La violencia en Argentina...", op. cit., Controversia $\mathrm{N}^{\circ} 2-3$, pp. 10-11.
} 
Una vez establecido en el país, el líder y la cúpula militar intentan ubicarse en un punto equidistante. Así lo refiere Bufano:

"A partir del 13 de julio el gobierno de Lastiri y posteriormente el de Perón se ubican fuera de la presunta lucha de fracciones. Como si el estado fuera un agente mediador y ajeno a la violencia cotidiana, se intenta mostrar una imagen que confundirá a amplios sectores del campo popular, incluyendo a capas medias progresistas: las derechas y las izquierdas se ultiman entre sí mientras el estado actúa como elemento pacificador. Perón exhorta al desarme y crea una oficina para recibir las armas que sólo la izquierda deberá entregar. Mientras tanto, el Ministerio de Bienestar Social acumula modernas ametralladoras belgas y norteamericanas." 14

A partir de mayo de 1973 la ofensiva militar pasó a la órbita del Estado, yendo de las detenciones a los asesinatos. Esta no fue la lectura de las organizaciones armadas, las cuales continuaron hacia una escalada violenta. Ni siquiera el retroceso de la dictadura detuvo la avanzada. En ese entonces, se produjo un desorden político y militar donde no estuvo claro el porqué del empleo de la violencia. En este fuego cruzado, exacerbado por el foquismo, los sectores progresistas retiraron su apoyo a las organizaciones armadas, de manera que se dio un movimiento de expulsión de la violencia armada hacia afuera de los márgenes tolerables por el orden social deseable.

Bufano plantea que las organizaciones armadas jugaban su suerte entre el militarismo y la falsa ofensiva. No obstante habían ganado un espacio importante entre los sectores obreros más combativos; pero el diagnóstico de situación era erróneo:

"La cuestión radica en que las formaciones políticas armadas de la Argentina de ese período confundieron la crisis del estado y el alza importante en la conciencia de algunos sectores obreros, con la descomposición general del sistema. Ese es el error global que hoy se califica como foquismo. Aún no se estaba en condiciones de discutir el poder a la burguesía; por el contrario los sectores monopólicos y su ejército se preparaban para su contraofensiva." ${ }^{\prime 15}$

\section{El golpe militar y el cambio de escenario...}

Terminado 1975 la situación de las organizaciones armadas era de un aislamiento consumado y una derrota que empezó a ser definitiva. Pero, a pesar de ello la actitud de Montoneros fue de ofensiva político-militar y de contemplar en todo momento una alternativa partidaria. Al respecto, Bufano refiere que:

"Se inicia entonces una campaña en contra del golpe que se avecina; pero nuevamente priman las concepciones ya no militares, sino militaristas, pues el propio Clausewitz (...), hubiera aconsejado un repliegue en orden. En cambio, la campaña para detener el golpe se basa en acciones espectaculares dirigidas contra las Fuerzas armadas; la intención es atemorizarlas contra una supuesta guerra civil en caso de golpe de estado. El efecto producido es inverso y se polarizan las fuerzas: ahora sí es una lucha entre aparatos que no acompaña ni está integrada a la lucha de clases."16

Vemos cómo la lucha entre burgueses y proletarios se desdibujaría en quienes se arrogaban ser los abanderados de cada sector. Y este planteo de lucha de aparatos constituirá el suelo para la llamada "teoría" de los dos demonios.

\footnotetext{
${ }^{14}$ Bufano, Sergio, "La violencia en Argentina...”, op. cit., Controversia $\mathrm{N}^{\circ} 2-3$, pp. 10-11.

15 Bufano, Sergio, "La violencia en Argentina...", op. cit., Controversia $\mathrm{N}^{\circ}$ 2-3, p. 11.

${ }^{16}$ Bufano, Sergio, "La violencia en Argentina...", op. cit., Controversia $\mathrm{N}^{\circ} 2-3, \mathrm{p} .11$.
} 
Sobre las acciones espectaculares no se dice mucho en el artículo en cuestión, pero tenían un lugar clave principalmente en el accionar de Montoneros. En lo que sí hace hincapié Bufano es que las acciones de las organizaciones insurgentes se contradecían con las consignas declamadas. Para ese entonces, el sector obrero ya estaba distante de la lucha armada.

El autor concluye su artículo señalando la falta de autocrítica de las organizaciones revolucionarias; y que la derrota sufrida dio lugar a que se confunda a la lucha armada con el terrorismo y la "violencia en general". Además se destaca el lugar alcanzado por la vanguardia revolucionaria de ese entonces.

A continuación tomaremos distancia del planteo de Bufano, próximo al marxismo, para introducir teorías que reformularán el problema de la violencia en otros términos. La misma es una perspectiva culturalista heredera del giro lingüístico, el posestructuralismo y el psicoanálisis.

\section{La violencia y lo social}

A partir de su análisis sobre el totemismo, Freud se preguntó de forma original por los sistemas religiosos y sociales. ${ }^{17}$ Es precisamente el tótem quien permite la organización de los grupos y el establecimiento de jerarquías. Esta cuestión también se lee con Durkheim en Las formas elementales de la vida religiosa. El sistema totémico en Australia (1912), donde también se interroga sobre qué hace posible la vida en sociedad y cómo se pone límites a la violencia. Este autor concluye que toda sociedad se constituye a partir de un conjunto de valores sagrados y de sentimientos compartidos que estarán en directa relación con las prohibiciones y leyes fundamentales.

Por su parte, Bataille señala que el esfuerzo por erradicar la violencia lleva a establecer prohibiciones, ya que el mundo del trabajo y la razón no bastan para absorber la violencia. Sin embargo hay algo que insiste... "Hay en la naturaleza, y subsiste en el hombre, un impulso que siembre excede los límites y que sólo en parte puede ser reducido. Por regla general, no podemos dar cuenta de ese impulso."

En la perspectiva de este autor se propone pensar lo social con un modelo energético, donde necesariamente se producen derroches y las prohibiciones tratan de poner límites. Esto evidencia que no habría continuidades armónicas, sino más bien rupturas que tratan de ser encauzadas por un orden determinado, pero que aun así resta un margen de azar e incertidumbre. Así lo refiere Bataille: “(...) en las prohibiciones esenciales vemos el rechazo que opone el ser a la naturaleza entendida como derroche de energía viva y como orgía del aniquilamiento (...)." Es justamente en la tensión entre ser y naturaleza donde se puede explorar el costado provechoso de este modelo, que tiene como fundamental la prohibición de la violencia (la muerte y la sexualidad).

Otra consideración que puede resultar controvertida de esta perspectiva es que la violencia reafirma el lazo social, porque: "La transgresión no es la negación de lo prohibido, sino que lo supera y lo completa". ${ }^{20}$ La violencia completa a lo prohibido porque le da su razón de ser y hace que se reactualice permanentemente; y supera a lo prohibido porque siempre hay algo no contemplado por la prohibición y porque la transgresión es de orden irracional.

Para el sostenimiento de las prohibiciones fundamentales resultan centrales, como señala Durkheim, los valores y sentimientos compartidos, que servirían de suelo común para erigir los lazos sociales. Sobre esto reflexiona Sergio Tonkonoff:

"Esta comprensión, si se la desprende de sus supuestos sociológicamente monistas y epistemológicamente realistas, tiene el mérito de poner en primer plano la irreductibilidad

\footnotetext{
${ }^{17}$ Freud, Sigmund, "El retorno infantil del totemismo", op. cit.

18 Bataille, Georges, El erotismo, op. cit., p. 44.

${ }^{19}$ Bataille, Georges, El erotismo, op. cit., p. 65.

${ }^{20}$ Bataille, Georges, El erotismo, op. cit., p. 67.
} 
de los afectos y las creencias colectivas y de su rol esencial en la producción y reproducción de lo social organizado, tanto como su transformación. Permite, finalmente, re-situar a las prácticas y los mecanismos de prohibición, transgresión y castigo en el espacio más concentrado de la cultura." 21

Ubicados ya en el terreno de la cultura, se puede arriesgar que todo orden socio-simbólico se constituye a partir de algún tipo de alteridad radical. ${ }^{22}$ La misma es puesta afuera de cada sociedad por parte de las prohibiciones, y para conjurar esa alteridad radical se emplea distinto tipo de puniciones.

Llegados a este punto, es posible sostener que la tensión entre lo social y la violencia puede ser desplegada en toda su densidad desde las teorías de la cultura; sólo así se puede dar cuenta de las prohibiciones estructurantes del orden cultural, de los afectos, la imaginación y los fantasmas que atraviesan, constituyen y exceden ese orden y sus sujetos.

\section{Lo abyecto como reactivo de lo social}

Siguiendo con el derrotero teórico que va de Freud a Bataille se llega a la conclusión de que la violencia es un elemento inerradicable y que constituye un afuera y un adentro de lo social. Una de las formas de ponerle límite a esa violencia es por medio del sacrificio. Esto se refleja en la producción de René Girard, donde se describe sociedades en las que el sacrificio institucionalizado tiene un papel central para procesar la violencia. Así lo plantea el autor francés:

"Existe (...) un denominador común de la eficacia sacrificial, tanto más visible y preponderante cuanto más viva permanece la institución. Este denominador es la violencia intestina; son las disensiones, las rivalidades, los celos, las peleas entre allegados lo que el sacrificio pretende ante todo eliminar, pues restaura la armonía de la comunidad y refuerza la unidad social. (...) Gracias a él [el sacrificio], las poblaciones permanecen tranquilas y no se agitan. Refuerza la unidad de la nación (...)."23

Esta referencia nos muestra el tratamiento que se le puede dar a la violencia al interior de un grupo para el sostenimiento del orden. El caso de la violencia hacia el exterior del grupo nos remite a la forma de resolución de conflictos por excelencia: la guerra. Ya lo planteó Freud en El malestar en la cultura y otros ensayos, dando cuenta de qué tipo de violencia es la que se practica: "El Estado combatiente se permite todas las injusticias y todas las violencias, que deshonrarían al individuo." 24

Esta cita nos invita a reflexionar sobre por qué se entendió como una guerra a los conflictos armados de la década de 1970 y sobre cómo operó el gobierno de facto de ese entonces.

También Bataille sigue un planteo similar y advierte sobre la regulación de la muerte a través de la guerra, la cual es un tipo de violencia organizada colectivamente. En esto, la transgresión organizada es fundamental, ya que forma con lo prohibido un complejo que delimita la vida social.

En las sociedades contemporáneas la forma del sacrificio retrocede y la guerra es contenida. Esto es posible por el imperio de la ley, que se pone por encima de todo poder, garantizando la paz. El sistema judicial racionaliza la violencia y el Estado tiene el monopolio de ella. En este planteo de

\footnotetext{
${ }^{21}$ Tonkonoff, Sergio, "Mito-lógicas...", op. cit., p. 6.

22 Para debates actuales sobre el asunto puede consultarse Mouffe, Chantal, En torno a lo politico, Fondo de Cultura Económica, Avellaneda, 2009; y Stavrakakis, Yannis, La izquierda lacaniana: psicoanálisis, teoría, política, Buenos Aires, Fondo de Cultura Económica, 2010.

${ }^{23}$ Girard, René, La violencia y lo sagrado, op. cit., p. 16.

${ }^{24}$ Freud, Sigmund, "Consideraciones de actualidad sobre la guerra y la muerte", op. cit., p. 101.
} 
Girard la violencia está en el centro del sistema pero es organizada respondiendo a un orden. Así lo refiere el autor:

"El sistema judicial aleja la amenaza de la venganza. No la suprime: la limita efectivamente a una represalia única, cuyo ejercicio queda confiado a una autoridad soberana y especializada en esta materia. Las decisiones de la autoridad judicial siempre se afirman como la última palabra de la venganza." 25

Vemos que la venganza tendría límite con las penas aplicadas por ley. Ese límite se logra con la violencia penal que, como señala Tonkonoff, tiene carácter de trascendente pues permite actualizar los fundamentos últimos que cohesionan a los grupos y les dan inteligibilidad.

Ahora bien, siempre hay un resto que insiste y persiste bajo distintas formas de violencia, por ejemplo la llamada justicia por mano propia, los casos de gatillo fácil y toda otra forma de disputa del monopolio de la fuerza al Estado.

Como ya se mencionó, la religiosidad domestica la violencia, la regula y la ordena, por ello todo otro invento social que cobre una forma similar a la religiosa servirá de cohesionador colectivo. En estos casos lo sagrado encierra una violencia contenida.

\section{Un modelo energético para entender la violencia}

Como vimos, el acuerdo extendido de las organizaciones armadas era que para hacerse del poder no quedaba otro camino que la violencia, incluso en el marco de gobiernos peronistas. Ahora bien, lo que diferencia el tipo de violencia de las organizaciones político-militares a primera vista es la legitimidad, los métodos empleados y los objetivos de su programa; en contraposición a la legitimidad, los métodos empleados y los objetivos del programa del Gral. Onganía y sus sucesores militares.

Un supuesto central del marxismo es que los conflictos de clase se resuelven por medio de la violencia. No obstante, como vimos con Bataille hay toda una serie de prohibiciones históricas que encausan la violencia, la cual persiste a través de los tiempos bajo distintas formas; y el conflicto nunca es resuelto del todo.

Como señala Bufano, en un primer momento la violencia debía asociarse con la justicia popular y la disputa de la hegemonía de la fuerza. Por ello, cada acción y cada muerte debían explicarse por sí mismas y obtener buena repercusión entre los trabajadores. Este será una espiral de violencia que irá creciendo y levantando gradualmente la prohibición de dar muerte. Se daba muerte y se entregaba la vida a la espera de producir nuevas prohibiciones que serían las propias de una sociedad socialista.

De acuerdo con Bufano, para 1973 la violencia ya estaba extendida en las prácticas de amplios sectores. Esto parece ser leído como un clima revolucionario por el marxismo de la época, pero aquí hemos planteado que lo que ocurría era el levantamiento de prohibiciones, un creciente estado de indeterminación y la lucha por el establecimiento de un nuevo orden.

El Estado no lograba pacificar los lazos sociales, y de esto sacó rédito el sector liderado por el Gral. Videla para hacer que las Fuerzas Armadas sean reclamadas y vistas como el único sector que podría ponerse por sobre los intereses de las facciones en conflicto. En este sentido, hubo un giro de la sociedad civil para expulsar fuera de los márgenes tolerables por el orden social a las organizaciones de izquierda. Ello combinado con la crisis de sentido que comenzaba a atravesar la lucha armada, la cual se iba quedando sin interlocutores ni consenso.

\footnotetext{
${ }^{25}$ Girard, René, La violencia y lo sagrado, op. cit., p. 23.
} 
Bufano advierte que un error clave era creer que estaban dadas las condiciones para disputar el poder a la burguesía, y que el sistema capitalista se encontraba en descomposición. Esta falla en el diagnóstico hizo que los avances en el terreno político recaigan en el militarismo sin su correlato en el terreno cultural.

Para introducir los problemas de la cultura, el marxismo debió revisar la metáfora base/superestructura, ${ }^{26}$ cuestión que no permeó en las organizaciones político-militares argentinas. Al respecto, Tonkonoff cita el aporte del estructuralismo:

"Pero Lévi-Strauss tiende a sustituir el clásico enfoque marxista del vínculo estructura (base) - superestructura para dar cuenta de esa mediación (lo real y sus imágenes). Propone, en cambio, el estudio de la relación entre las formas: primero de las "separaciones diferenciales" de las formas entre sí y luego del modo en que estas separaciones se agrupan, se combinan, se oponen." 27

Los sistemas de significación quedarían instituidos ya no con la preeminencia de la base económica, sino a partir de prohibiciones -límites-, exclusiones fundantes, lo cual resulta en la delimitación del ámbito de lo social en términos de ordenamientos simbólicos que producen visibilidad y constricción al mismo tiempo.

Girard plantea que: "La violencia constituye el auténtico corazón y el alma secreta de lo sagrado", ${ }^{28}$ lo cual nos indica que la violencia de las organizaciones armadas debió investirse de cierto alo de veneración y respeto para tener mayor eficacia. Esto tendería puentes con los sentidos míticos -que preocupaban tanto a Freud como a Lévi-Strauss- que pueden devenir colectivos e inconscientes. Ya en el terreno de la afectividad... "Se trata de relatos narrados según la lógica del pensamiento primario que, socializados ritualmente, proveen de marcos cognitivos intensamente penetrados de afectividad e imaginación". ${ }^{29}$

Por último, una de las preguntas que quedan abiertas es sobre los sentidos de la muerte en la década de 1970. Sobre ello, Bárbara Ohanian plantea que:

"El sentido sacrificial que recubrió las muertes que resultaron de la disposición a ser muerto tenía una eficacia posible, que era la del nacimiento de un nuevo orden, sea el socialismo, sea la patria socialista, etc. De hecho, nada fue lo mismo luego de tantos sacrificios y muertes. Sin embargo, lo que nació no fue lo que se esperaba que fuera el resultado de esos sacrificios." 30

Lo que sí puede concluirse es que la eliminación de la alteridad nunca puede ser concretada del todo, y por ello emergen del fuego cruzado tensiones en la significación que llegan hasta nuestros días.

26 Véase por ejemplo: Volóshinov, Valentín [1929], El marxismo y la filosofía del lenguaje, Madrid, Alianza, 1992; y Althusser, Louis, Ideología y aparatos ideológicos del Estado, Buenos Aires, Nueva Visión, 1970.

${ }^{27}$ Tonkonoff, Sergio, "Intercambio de tiros y tráfico de drogas...", op. cit., p. 464.

${ }^{28}$ Girard, René, La violencia y lo sagrado, op. cit., p. 38.

${ }^{29}$ Tonkonoff, Sergio, "Mito-lógicas...”, op. cit., p. 16.

30 Ohanian, Bárbara, "Muerte y sacrificio. Algunas reflexiones a partir del debate «del Barco»", en línea en http://jornadasjovenesiigg.sociales.uba.ar/eje-13-genocidio-memoria-derechos-humanos/, fecha de consulta: 21/05/2013, p. 12. 


\section{Bibliografía}

Bataille, Georges [1957], El erotismo, Barcelona, Tusquets, 1998.

Freud, Sigmund [1913], "El retorno infantil del totemismo", en Tótem y Tabú, Madrid, Alianza, 1985.

Freud, Sigmund [1915], "Consideraciones de actualidad sobre la guerra y la muerte", en El malestar en la cultura, Madrid, Alianza, 1985.

Gago, Verónica, Controversia: una lengua del exilio, Ediciones Biblioteca Nacional, Quilmes, 2012.

Gillespie, Richard [1982], Soldados de Perón: historia crítica sobre los Montoneros, Villa Ballester, Sudamericana, 2011.

Girard, René [1977], La violencia y lo sagrado, Barcelona, Editorial Anagrama, 1995.

Ohanian, Bárbara, "Muerte y sacrificio. Algunas reflexiones a partir del debate «del Barco»", en línea en http://jornadasjovenesiigg.sociales.uba.ar/eje-13-genocidio-memoria-derechoshumanos/, fecha de consulta: 21/05/2013.

Tonkonoff, Sergio, "Intercambio de tiros y tráfico de drogas: etnocriminología en Río de Janeiro", en Alegatos. Revista del Departamento de Derecho de la División de Ciencias Sociales y Humanidades $\mathrm{N}^{\circ}$ 58, Azcapotzalco, Universidad Autónoma Metropolitana-Azcapotzalco, 2004.

Tonkonoff, Sergio, "Mito-lógicas. La cuestión criminal en el centro de la cultura", en Mariano Gutiérrez (comp.), Populismo punitivo y justicia expresiva, Buenos Aires, Di Plácido Editores, 2011.

\section{Hemeroteca}

Revista Controversia para el examen de la realidad argentina [1979-1981], edición facsimilar, Buenos Aires, Ejercitar la Memoria editores, 2009. 